$5 \quad$ Lun $^{\mathrm{a}, \mathrm{d} *}$

6 $\operatorname{Lun}^{\mathrm{a}, \mathrm{d} *}$ (1)

$7 \mathrm{UK}$

\title{
The association between Toxoplasma gondii infection and
}

postpartum blues

Jiang-Mei Gao ${ }^{\text {a\#}}$, Zhi-Hui He ${ }^{\text {b\# }}$, Yi-Ting Xie ${ }^{\mathrm{a}, \mathrm{c}}$, Geoff Hide ${ }^{\mathrm{d}}$, De-Hua Lai ${ }^{\mathrm{a} *}$, Zhao-Rong

${ }^{\mathrm{a} C e n t e r}$ for Parasitic Organisms, State Key Laboratory of Biocontrol, School of Life Sciences, and Key Laboratory of Tropical Disease Control of the Ministry of Education, Zhongshan School of Medicine, Sun Yat-Sen University, Guangzhou 510275, China;

${ }^{b}$ Department of Obstetrics and Gynecology, the First Affiliated Hospital of

Guangzhou Medical University, Guangzhou510120, China;

'Department of Human Parasitology, School of Basic Medical Science, Hubei

University of Medicine, Shiyan 442000, China.

5 Biomedical Research Centre and Ecosystems and Environment Research Centre,

6 School of Environment and Life Sciences, University of Salford, Salford, M5 4WT,

$8 \quad$ \# These authors contributed equally.

*Corresponding authors: laidehua@mail.sysu.edu.cn (D.H.L) or

1sslzr@mail.sysu.edu.cn (Z.R.L.) 


\section{Abstract}

Introduction and Aim: Toxoplasma gondii is an intracellular protozoan parasite infecting approximately $30 \%$ of the global human population. It has often been suggested that chronic infection with $T$. gondii is related to personality changes and various mental disorders including depression. It is not known whether this includes post-partum blues or depression. In this study, we test the hypothesis that there is a relationship between $T$. gondii infection and post-partum blues by measuring the association between infection and postpartum blues.

Methods: A total of 475 Chinese women who have just given birth were detected serology for Toxoplasma $\operatorname{IgG}$ and IgM antibodies, and evaluated the degree of depression by Hamilton Depression Scale (HAMD) score. Data were analyzed by Chi-square or Fisher's Exact tests using SPSS software.

Results: We found an overall Toxoplasma seroprevalence of 5.68\% (27/475; 95\% CI: 3.59 - 7.77) which was broken down into a prevalence of $6.60 \%(7 / 106 ; 95 \%$ CI: 1.80 - 11.41) in mothers with post-partum blues and 5.42\% (20/369; 95\% CI: $3.10-7.74)$ in non-affected mothers. There was no significant association between infection and post-partum blues $(p=0.64)$.

Conclusion: The results suggest that there is no relationship between $T$. gondii infection and postpartum blues, at least in this sample of patients from China.

Key words: Toxoplasma gondii, postpartum blues, seroprevalence, newborn, puerpera, Hamilton Depression Scale 


\section{Introduction}

Toxoplasma gondii, which infects $30 \%$ to $50 \%$ of the global population, is one of the most common parasites affecting both healthy and immunocompromised humans (Furtado et al., 2011; Klaren and Kijlstra, 2002; Prandota, 2014). It is believed that $T$. gondii infection, termed toxoplasmosis, in immunocompetent individuals is generally considered asymptomatic (Halonen and Weiss, 2013). However, considering an extended set of disease conditions, evidence is accumulating that strongly suggests that this parasite may be implicated in neurodegenerative diseases and are gradually emerging as a global health threat (Nissapatorn, 2010; Furtado et al., 2011; Torgerson and Mastroiacovo, 2013). For example, chronic infection with T. gondii is more frequent in individuals with schizophrenia than in psychiatrically healthy controls, as indicated in several studies from different countries (Torrey and Yolken, 2001, 2003; Torrey et al., 2007). In addition, a number of studies have also demonstrated that $T$. gondii seropositivity is related to personality changes and various mental disorders including development of suicidal tendencies, obsessive compulsive disorder, bipolar disorder, and depression (Sutterland et al., 2015; Arling et al., 2009; Hinze-Selch et al., 2010; Ling et al., 2011; Okusaga et al., 2011; Tedla et al., 2011).

Depressive disorder, also known as clinical depression, is a mood disturbance characterized by changes in mood, and loss of interest, pleasure, cognitive function, sleep, appetite, or energy level (Pratt and Brody, 2008). Furthermore, clinical depression is commonly associated with significant morbidity and mortality (Hsu et al., 2014). It has been reported that, during 2009-2012, about 7.6\% Americans aged 12 and over had 
depression (Pratt and Brody, 2014). However, the prevalence of mood disorders in China appears to be lower than that found in North America and Western Europe. Data indicated that the prevalence of major depressive disorders in China was at $1.96 \%$ in Kunming, $5.3 \%$ in Beijing, 3.6\% in Shenyang and $1.15 \%$ in Jiangsu, respectively (Hu, 2003; Lu et al., 2008; Ma et al., 2009; Qin et al., 2008). Moreover, depression is also considered a common mental health problem among women of childbearing age, with a higher prevalence rate ranging from 10 to 32\% (Ertel et al., 2011; Wang et al., 2011). Postpartum nonpsychotic depression is the most common complication of childbirth, affecting approximately $10-15 \%$ of women, and represents a considerable health problem affecting women and their families. A mother's ongoing depression can contribute to later emotional, behavioral, cognitive and interpersonal problems in the offspring. Because of these serious consequences, exploring potential factors, early diagnosis and intervention treatment of postpartum illnesses are imperative for the health and well-being of mother and child (Robertson et al., 2004). Previous studies have consistently demonstrated common significant predictors of postpartum depression as follows: experiencing depressed moods or anxiety during pregnancy, life events, no social support and socioeconomic status (Patel et al., 1999; Bartley, 1994; Beck, 2001; Brugha et al., 1998; Neter et al., 1995). Furthermore, studies have shown that latent $T$. gondii infection is associated with symptoms of depression during pregnancy (Groer et al., 2011). Post partum blues are self-limiting depression commonly found within one week soon after delivery, and are considered as important indicators of depression (Maliszewska et al., 2016; Reck et al., 2009). However, it remains unknown whether 
parasite infection may play an important role in postpartum blues. We are interested in the relationship between chronic latent $T$. gondii infection and the development of postpartum blues in new mothers. Using a cohort of new mothers in China, we aim to investigate this globally important question and to test the hypothesis that $T$. gondii infection may be more frequent and/or more intense in patients with major postpartum depression compared with psychiatrically healthy controls.

\section{Materials and Methods}

\section{Participants and Questionnaires}

In this study, blood samples were collected from 475 women one week after delivery (puerpera) in the First Affiliated Hospital of Guangzhou Medical University, China. Mothers were randomly selected for this study and all those selected were tested by a psychiatrist irrespective of any obvious signs of depression. Due to the low prevalence of Toxoplasma infection in China, the sample size needed to ensure sufficient statistical power (minimum $\mathrm{n}=442$ ) was calculated using a previously published prevalence of 7.8\% (ONIHPDCSS, 2005). Furthermore, this value concurred with our previous studies (collated from greater than 120000 pregnant women (1990 - 2010)) that recorded the seroprevalence of Toxoplasma to be less than $10 \%$ in this demographic group (Gao et al., 2012). Sera were separated by centrifugation and stored at $-80^{\circ} \mathrm{C}$ until serological testing. All participant information was obtained (usually 2-3 days postpartum) through questionnaires and 
recorded. These included participants' age, occupation, relevant eating habits

(consumption of raw or undercooked meat - past and current) and cat contact (current and past cat ownership, cats in the same household, playing closely with cats, cleaning cat litter).

\section{Ethical Approval}

This study was approved by the Medical Science Ethical Committee of Sun-Yet San University and the First Affiliated Hospital of Guangzhou Medical University. All enrolled participants were informed about the objectives of the study, and written informed consent was obtained from all of them.

\section{Hamilton Depression Scale (HAMD) score}

Psychiatrists evaluated the degree of depression according to the HAMD

(Hamilton, 1967) depression scale score, clinical symptoms and the exclusive criteria.

A HAMD score of $<8$, was considered normal; 8-19, indicated mild depression; 20-

34 , indicated medium depression; and $\geq 35$, was considered as severe depression. All patients with mild, medium and severe depression were considered as postpartum blues cases according to their clinical signs.

\section{Serological Tests}

All serum samples were tested for anti-T. gondii antibodies - both IgM (indicator of acute infection) and IgG (indicator of chronic infection) using a commercially available 
enzyme-linked immunosorbent assay (ELISA) kit (Modern Gaoda Biotechnology

$$
\text { Company, Beijing) according to the manufacturer's instructions (accuracy }>96.7 \% \text {, }
$$
detection limit of $5 \mathrm{IU} / \mathrm{ml}$ and coefficient of variation $<15 \%$.). Positive, negative and cutoff serum controls were included in every plate. The optical densities (ODs) were measured at $450 \mathrm{~nm}$ in a microplate reader (Thermo Scientific Multiskan FC, Thermo Scientific, China). All serum samples were run in triplicate. Samples with ODs above the cutoff value were considered as serologically positive.

\section{Statistical Analysis}

Data on the prevalence of anti-T. gondii antibodies and depression symptoms in population groups were analyzed by Chi-square or Fisher's Exact tests using SPSS software. Risk factors of occupation, serolopositivity for $T$. gondii, raw meat consumption and a significant contact with cats were evaluated by using odds ratios (ORs), together with their corresponding 95\% confidence intervals (95\% CIs). Bias corrected ORs were obtained by adding 0.5 to each data point.

\section{Results}

The collection of 475 serum samples taken from the new mothers was examined for anti-T. gondii IgG and IgM antibodies using enzyme-linked immunosorbent assay (ELISA). All 475 puerpera samples were IgM negative, suggesting that no current infection of the parasite was occurring. Anti-Toxoplasma positive IgG was detected in 
27 (5.68\%; 95\% CI: 3.59 - 7.77) of the 475 serum samples as shown in Table 1. Hamilton Depression Scale scores in the same set of new mothers were determined. All patients with mild, medium and severe depression were considered as true cases having postpartum blues according to their clinical signs. There was no significant association between $T$. gondii seropositivity and postpartum blues ( $\mathrm{p}=$ $0.643 ; \mathrm{n}=475)$. When broken down, the seroprevalence of $T$. gondii was slightly higher in the new mothers with postpartum blues $(6.60 \%, 7 / 106 ; 95 \% \mathrm{CI}: 1.80-$ 11.41) than that in the remaining normal group $(5.42 \%, 20 / 369 ; 95 \% \mathrm{CI}: 3.10-7.74)$ but this was not significant $(p=0.643, \mathrm{OR}=1.234[0.507-3.002])-$ see Table 1 .

To further explore the hypothesis that $T$. gondii infection is related to postpartum depression, it is possible that there is a quantitative influence on the relationship. For example, T. gondii-positive new mothers might show higher scores on the depression scale when compared to their uninfected counterparts. To examine that, we further analyzed the relationship between $T$. gondii immunoglobulin G (IgG) optical densities (ODs) and the Hamilton Depression Scale (HAMD) scores. We found that depression scores were not significantly different $(p=0.873)$ between the positive and negative T. gondii optical density (ODs) groups in the entire population of new mothers ( $\mathrm{n}=475$ ) (Figure S1A). In addition, as shown in Figure S1B, the T. gondii positive group $(\mathrm{n}=27)$ did not show a positive correlation of $T$. gondii ODs (serointensity) with the Hamilton Depression Scale (HAMD) scores $(p=0.214)$. Although we recognize that the sample sizes in this breakdown are small, these analyses further 
support the prevalence data that suggests there is no significant association between infection and postpartum blues.

According to previous reports, the probability of becoming infected with $T$. gondii increases with age because the infection is ubiquitous and the probability of being exposed to infection increases with age (Hinze-Selch et al., 2007). Therefore, we analysed the seroprevalence of $T$. gondii in the 475 pregnant women in different age groups (Table 2) and the results demonstrated that there was no significant correlation between $T$. gondii seroprevelence and age $(p=0.872)$. Therefore, the results from our current study demonstrate that anti- $T$. gondii seropositivity is not age dependent in this sample of mothers. One possible reason for the results is that the age range of pregnant women is too narrow to reveal this effect and we also recognize that the sample sizes in this breakdown are small. We also found that the incidence of postpartum blues did not show a significant relationship with age of the mothers $(p=$ 0.610) (Table 2) although again our numbers of individuals were small. To investigate whether there was any association between the prevalence of either post-partum blues or T. gondii seropositivity, analyses were conducted on a breakdown of occupational status of the participants based on the questionnaire data (Table 3). The participants were categorized into 10 occupational type groups: farmers (most are less well educated and live in rural areas), workers (born in the city, most are less well educated and tend to be employed in factories and companies), medical staff (well-educated, with a good understanding of health and hygiene), 
managers (born in the city and most are well-educated but may not have an understanding of health issues), teachers (well-educated, have health awareness but may not have detailed knowledge), business staff (may or may not be well-educated, most of them do not have an understanding of health issues), self-employed (may or may not be well-educated, most of them do not have an understanding of health issues), housewives (most are less well educated and are responsible for the routine work in the family), unemployed (most are less well educated and have been out of work for the past few years) and others (no details of their working status). There was no significant difference in anti-T. gondii seroprevalence $(p=0.971)$ and incidence of postpartum blues $(p=0.918)$ between the different occupational groups. We again recognize that there are small numbers of individuals sampled in this analysis.

We also investigated traditional risk factors associated with the seroprevalence of T. gondii and prevalence of postpartum blues in new mothers (Table 4). Surprisingly, our analyses showed that contact with cats and consumption of raw or uncooked meat, two generally well established risk factors, were not associated with T. gondii seropositivity ( $p=0.766$ and 0.357 , respectively). By contrast, consumption of raw or uncooked meat was significantly associated with increased prevalence of postpartum blues among new mothers $(p=0.007)$ albeit based on small numbers. The prevalence of postpartum blues in the group of participants that consumed raw meat was significantly higher than the group that did not consume raw meat $(32.97 \%$ [30/91] vs $19.79 \%$ [76/394], OR: 1.993, 95\% CI: 1.204-3.229). In addition, the association between cat contact and prevalence of postpartum blues in new mothers is 
close to significance at $p=0.061$ suggesting that a larger sample size would be useful to explore this further. The prevalence in the cat-contact group $(29.47 \%, 28 / 95)$ is slightly higher than that of the non-cat-contact group $(20.53 \%, 78 / 380$; OR: 1.618 ; 95\%CI: 0.975-2.685).

\section{Discussion}

Depression during the perinatal period does not only have a significant impact on quality of life of the mother (Darcy et al., 2011), but also influences the developmental outcomes of their children (Ertel et al., 2011; Turney, 2012). Many previous studies have tried to identify the role of $T$. gondii in general neurological and psychiatric conditions in humans (Torrey et al., 2007; Arling et al., 2009; Groer et al., 2011; Hinze-Selch et al., 2010; Prandota, 2014; Sutterland et al., 2015). Clear associations seem to be emerging for schizophrenia (Torrey et al., 2007, Sutterland et al., 2015) and probable associations with attempted suicide (Arling et al., 2009) and personality disorders (Hinze-Selch et al., 2010). A major detailed meta-analysis provided convincing evidence of an association between $T$. gondii infection and schizophrenia but also suggested links with bipolar disorder, obsessive-compulsive disorder, addiction but not for major depression (Sutterland et al., 2015). Several other studies also demonstrated no link with major depression (Gale et al 2014; Gale et al 2016; Suvisaari et al 2017). Some studies have focused specifically on prenatal depression. Groer et al (2011) conducted a study on women during pregnancy and showed that there was no significant difference in T. gondii seroprevalence between 
mothers with prenatal depression and controls. However, they observed that higher titers of $T$. gondii IgG antibody were positively correlated with increased measures of depression and anxiety (as measured by the Profile of Mood States -POMS method). Furthermore, other studies have shown no association between the seroprevalence of $T$. gondii and prenatal depression in both low (5.25\%) (AlvaradoEsquival et al., 2017) and in high (59\%) (Nourollahpour Shiadeh et al., 2016) seroprevalence of infection. The latter study demonstrated that there was an association of increasing $T$. gondii IgG titer with increasing depression score (as measured by the Iranian version of the Edinburgh Post-Partum Depression Scale (EPDS) (Nourollahpour Shiadeh et al., 2016) however, no such association was found in the former one (EPDS, Mexican version) (Alvarado-Esquival et al., 2017). The consensus seems to be emerging that there is no association of $T$. gondii seroprevalence and prenatal depression although conflicting evidence exists with regard to association between serointensity and depression severity score. To our knowledge, no studies have been conducted that investigate any possible association between $T$. gondii infection and postpartum depression or postpartum blues, the latter being considered as sa elf-limiting mild depression commonly found in new mothers a few days after delivery (Maliszewska et al., 2016). In our study, we report the first investigation addressing this question. Using a cohort of 475 participants, we analyzed the association between $T$. gondii infection and postpartum blues. Our results show clearly that overall there was no significant association between seropositivity of T. gondii and new mothers with postpartum blues. This lack of 
association, between seroprevalence and postpartum blues, follows the same pattern revealed by studies on prenatal depression during pregnancy, described above, and supports a consensus that there may be no general association with perinatal depression. Furthermore, when we broke our data down further, we found no evidence of association between increasing $\operatorname{IgG}$ titer and depression severity score (as measured by the Hamilton Depression Scale). Although we do recognize that the low prevalence of $T$. gondii infection and prevalence of postpartum blues in our cohort reduces the power of such a detailed analysis. Again, though, our data, on the quantitative association between $T$. gondii IgG titer and depression score, is consistent with some other studies (Alvarado-Esquival et al., 2017). However, both our results and those of Alvarado-Esquival et al. (2017) conflict with those of Nourollahpour Shiadeh et al. (2016) on this quantitative association. This could be due, in part, to differences in either depression scoring methods or due to differing backgrounds of the prevalence of parasite infections. The former is unlikely, since both the studies of Alvarado-Esquival et al (2017) and Nourollahpour Shiadeh et al (2016) used variants of the EPDS. However, the background of prevalences in each study differed considerably (5.25\% and 59\%) with the former corresponding to our reported prevalence in our cohort (5.68\%) suggesting that this could be a factor. In China, the seroprevalence of $T$. gondii in the Chinese population $(\sim 10 \%)$ is much lower than that in some parts of South America and Europe (50\%-80\%) (Fromont et al., 2009; ONIHPDCSS, 2005). In pregnant mothers in China, the prevalence is also low ( $\sim 10 \%)$ and corresponds to the general population levels (Gao et al., 2012). In this 
study, the prevalence followed the typical low level found in China (5.68\%). The correspondence between the background prevalence in the study of AlvaradoEsquival et al (2017) and our study, both finding no quantitative association between T. gondii IgG titer and depression scores, supports a view that the conflict with the study of Nourollahpour Shiadeh et al (2016) could be related to prevalence. This also raises the issue that studies conducted in areas of low parasite prevalence may need substantially larger samples sizes to achieve the required power to conduct more detailed breakdowns of interactions. The question is clearly complex and it is possible that other interacting factors may confound any role that $T$. gondii may possess in perinatal depression.

If $T$. gondii infection is not the main factor causing postpartum blues nor depression in new mothers, are there any other factors, such as age, which could influence the seroprevalence of $T$. gondii or baby blues. Seroprevalence of $T$. gondii has been shown to that increase with age (Hinze-Selch et al., 2007). For instance, in the Israeli population, the seroprevalence rate of $T$. gondii is $7.6 \%$ in the $10-19$ years group, $31.4 \%$ in the $50-59$ years group, followed by a sharp increase to $58.1 \%$ in the $\geq 60$ years group (Markovich et al., 2014). In addition, similar results have been observed in pregnant women in Poland, where mean prevalence of IgG antibodies was seen at $40.6 \%$ and increased with age with a yearly seroconversion rate of $0.8 \%$ (95\% CI: 0.6-1.0, $p<0.001$ ) (Nowakowska et al., 2014). However, in an older study, Ye and Zou (1993) reported that seroprevalence of T. gondii in new mothers in China did not increase with age. In our study of Chinese new mothers, no age prevalence 
increase was observed $(p=0.610)$. These latter studies may be explained by bias due to a relatively narrow age window found in cohorts of new mothers/women in pregnancy.

In our study, we investigated the influence of occupation, association with cats and consumption of raw meat on the relationship between $T$. gondii infection and postpartum blues. Our data shows no significant association between these risk factors and infection or postpartum blues. However, we recognise that once our data was broken down to these levels of detail, sample sizes were small and future studies may be needed with increased statistical power to dissect these questions.

To our knowledge, this is the first study that has investigated the association of T. gondii infection with postpartum blues in new mothers. We found no association between the seroprevalence of T. gondii and clinically depressed participants. Other studies, that have focused on prenatal depression during pregnancy, have also failed to detect significant association with $T$. gondii seroprevalence. We propose, therefore, that, the current studies are tending towards a consensus that shows little support for the involvement of this parasite in perinatal depression in general. However, perinatal depression has a complex etiology and future larger scale studies may be required to unpick further detail and to investigate specific epidemiological interactions.

\section{Authors' contributions}

Jiang-Mei Gao and Zhao-Rong Lun they were responsible for designed research; Jiang-Mei Gao and Yi-Ting Xie conducted research; Zhi-Hui He provided essential 
materials; De-Hua Lai conducted analyzed data or performed statistical analysis; Jiang-Mei Gao, Geoff Hide, De-Hua Lai and Zhao-Rong Lun wrote paper.

\section{Funding}

This work was supported by the National Key R\&D Program of China (2017YFD0500400), National Natural Science Foundation of China (31772445) and the 111 project (B12003).

\section{Acknowledgments:}

The authors wish to thank the funding agencies and a cohort of Chinese new mothers.

\section{Conflict of interest}

The authors report no conflict of interest.

\section{References:}

Alvarado-Esquivel, C., Martínez-Martínez, A.L., Sánchez-Anguiano, L.F., Hernández-Tinoco, J., Castillo-Orona, J.M., Salas-Martínez, C., et al., 2017. Lack of association between Toxoplasma gondii exposure and depression in pregnant women: a case-control study. BMC. Infect. Dis. 17(1), 190.

Arling, T.A., Yolken, R.H., Lapidus, M., Langenberg, P., Dickerson, F.B., Zimmerman, S.A., et al., 2009. Toxoplasma gondii antibody titers and history of 
suicide attempts in patients with recurrent mood disorders. J. Nerv. Ment. Dis. 197, 905-908.

Bartley, M.,1994. Unemployment and ill health: understanding the relationship. J. Epidemiol. Community. Health. 48, 333-337.

Beck, C.T., 2001. Predictors of postpartum depression: an update. Nurs. Res. 50, 275285.

Brugha, T.S., Sharp, H.M., Cooper, S.A., Weisender, C., Britto, D., Shinkwin, R., et al.,1998. The Leicester 500 Project. Social support and the development of postnatal depressive symptoms, a prospective cohort survey. Psychol. Med. 28, 63-79.

Darcy, J.M., Grzywacz, J.G., Stephens, R.L., Leng, I., Clinch, C.R., Arcury, T.A., 2011. Maternal depressive symptomatology: 16-month follow-up of infant and maternal health-related quality of life. J. Am. Board. Fam. Med. 24, 249-257.

Ertel, K.A., Rich-Edwards, J.W., Koenen, K.C., 2011. Maternal depression in the United States: nationally representative rates and risks. J. Womens. Health (Larchmt). 20, 1609-1617.

Fromont, E.G., Riche, B., Rabilloud, M., 2009. Toxoplasma seroprevalence in a rural population in France: detection of a household effect. Bmc. Infect. Dis. 9, 76.

Furtado, J.M., Smith, J.R., Belfort, R.J., Gattey, D., Winthrop, K.L., 2011 Toxoplasmosis: a global threat. J. Glob. Infect. Dis. 3, 281-284.

Gale, S.D., Berrett, A.N., Brown, B., Erickson, L.D., Hedges, D.W., 2016. No association between current depression and latent toxoplasmosis in adults. Folia. 
379 Gale, S.D., Brown, B.L., Berrett, A., Erickson, L.D., Hedges, D.W., 2014. Association between latent toxoplasmosis and major depression, generalised anxiety disorder and panic disorder in human adults. Folia. Parasitol (Praha). 61(4), 285-292.

Gao, X.J., Zhao, Z.J., He, Z.H., Wang, T., Yang, T.B., Chen, X.G., et al., 2012. Toxoplasma gondii infection in pregnant women in China. Parasitology. 139(2), 139-147.

Groer, M.W., Yolken, R.H., Xiao, J.C., Beckstead, J.W., Fuchs, D., Mohapatra, S.S., et al., 2011. Prenatal depression and anxiety in Toxoplasma gondii-positive women. Am. J. Obstet. Gynecol. 204, 431-433.

Halonen, S.K., Weiss, L.M., 2013. Toxoplasmosis. Handb. Clin. Neurol. 114, $125-$ 145.

Hamilton, M., 1967. Development of a rating scale for primary depressive illness. Br. J. Soc. Clin. Psychol. 6(4), 278-296.

Hinze-Selch, D., Daubener, W., Eggert, L., Erdag, S., Stoltenberg, R., Wilms, S., 2007. A controlled prospective study of toxoplasma gondii infection in individuals with schizophrenia: beyond seroprevalence. Schizophr. Bull. 33(3), 782-788.

Hinze-Selch, D., Daubener, W., Erdag, S., Wilms, S., 2010. The diagnosis of a personality disorder increases the likelihood for seropositivity to Toxoplasma gondii in psychiatric patients. Folia. Parasitol (Praha). 57, 129-135. 
Hsu, P.C., Groer, M., Beckie, T., 2014. New findings: depression, suicide, and Toxoplasma gondii infection. J. Am. Assoc. Nurse. Pract. 26, 629-637.

Hu Bin, X.L.B.W., 2003. Epidemiological survey of depression prevalence in Jiangxi Province. Chin. J. Psychiatry. 36, 242-245.

Klaren, V.N., Kijlstra, A., 2002. Toxoplasmosis, an overview with emphasis on ocular involvement. Ocul. Immunol. Inflamm. 10, 1-26.

Ling, V.J., Lester, D., Mortensen, P.B., Langenberg, P.W., Postolache, T.T., 2011. Toxoplasma gondii seropositivity and suicide rates in women. J. Nerv. Ment. Dis. $199,440-444$.

Lu, J., Ruan, Y., Huang, Y., Yao, J., Dang, W., Gao, C., 2008. Major depression in Kunming: prevalence, correlates and co-morbidity in a south-western city of China. J. Affect. Disord. 111, 221-226.

Ma, X., Xiang, Y.T., Cai, Z.J., Li, S.R., Xiang, Y.Q., Guo, H.L., et al., 2009. Prevalence and socio-demographic correlates of major depressive episode in rural and urban areas of Beijing, China. J. Affect. Disord. 115, 323-330.

Markovich, M.P., Shohat, T., Riklis, I., Avni, R., Yujelevski-Rozenblit, D., Bassal, R., et al., 2014. Seroepidemiology of Toxoplasma gondii infection in the Israeli population. Epidemiol. Infect. 142, 149-155.

Maliszewska K, Świątkowska-Freund M, Bidzan M, Preis K., 2016. Relationship, social support, and personality as psychosocial determinants of the risk for postpartum blues. Ginekol Pol. 2016;87(6):442-7.

Neter, E., Collins, N.L., Lobel, M., Dunkel-Schetter, C., 1995. Psychosocial 
predictors of postpartum depressed mood in socioeconomically disadvantaged women. Womens. Health. 1, 51-75.

Nourollahpour Shiadeh, M., Rostami, A., Pearce, B.D., Gholipourmalekabadi, M., Newport, D.J., Danesh, M., et al., 2016. The correlation between Toxoplasma gondii infection and prenatal depression in pregnant women. Eur. J. Clin. Microbiol. Infect. Dis. 35(11), 1829-1835. Epub 2016 Aug 8. PubMed PMID: 27502929.

Nowakowska, D., Wujcicka, W., Sobala, W., Spiewak, E., Gaj, Z., Wilczynski, J., 2014. Age-associated prevalence of Toxoplasma gondii in 8281 pregnant women in Poland between 2004 and 2012. Epidemiol. Infect. 142, 656-661.

Okusaga, O., Langenberg, P., Sleemi, A., Vaswani, D., Giegling, I., Hartmann, A.M., et al., 2011. Toxoplasma gondii antibody titers and history of suicide attempts in patients with schizophrenia. Schizophr. Res. 133, 150-155.

ONIHPDCSS. 2005. A national survey on current status of the important parasitic diseases in human population. Zhongguo Ji Sheng Chong Xue Yu Ji Sheng Chong Bing Za Zhi. 23, 332-340.

Patel, V., Araya, R., de Lima, M., Ludermir, A., Todd, C., 1999. Women, poverty and common mental disorders in four restructuring societies. Soc. Sci. Med. 49, $1461-1471$.

Phillips, M.R., Zhang, J., Shi, Q., Song, Z., Ding, Z., Pang, S., et al., 2009. Prevalence, treatment, and associated disability of mental disorders in four provinces in China during 2001-05: an epidemiological survey. Lancet. 13, 
Prandota, J., 2014. Possible link between Toxoplasma gondii and the anosmia associated with neurodegenerative diseases. Am. J. Alzheimers. Dis. Other. Demen. 29, 205-214.

Pratt, L.A., Brody, D.J., 2014. Depression in the U.S. household population, 20092012. NCHS. Data. Brief. 1-8.

Pratt, L.A., Brody, D.J., 2008. Depression in the United States household population, 2005-2006. NCHS. Data. Brief. 1-8.

Qin, X., Wang, W., Jin, Q., Ai, L., Li, Y., Dong, G., et al., 2008. Prevalence and rates of recognition of depressive disorders in internal medicine outpatient departments of 23 general hospitals in Shenyang, China. J. Affect. Disord. 110, 46-54.

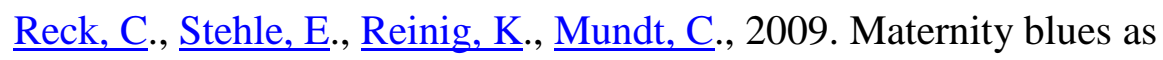
a predictor of DSM-IV depression and anxiety disorders in the first threemonths postpartum. J. Affect. Disord. 113(1-2), 77-87.

Robertson, E., Grace, S., Wallington, T., Stewart, D.E., 2004. Antenatal risk factors for postpartum depression: a synthesis of recent literature. Gen. Hosp. Psychiatry. 26, 289-295.

Sutterland, A.L., Fond, G., Kuin, A., Koeter, M.W., Lutter, R., van Gool, T., et al., 2015. Beyond the association. Toxoplasma gondii in schizophrenia, bipolar disorder, and addiction: systematic review and meta-analysis. Acta. Psychiatr. 
467 Suvisaari, J., Torniainen-Holm, M., Lindgren, M., Härkänen, T., Yolken, R.H., 2017.

468 Toxoplasma gondii infection and common mental disorders in the Finnish

469 general population. J. Affect. Disord. 223, 20-25.

470 Tedla, Y., Shibre, T., Ali, O., Tadele, G., Woldeamanuel, Y., Asrat, D., et al., 2011.

471 Serum antibodies to Toxoplasma gondii and Herpesvidae family viruses in

472 individuals with schizophrenia and bipolar disorder: a case-control study. Ethiop.

$473 \quad$ Med. J49, 211-220.

474 Torgerson, P.R., Mastroiacovo, P., 2013. The global burden of congenital

475 toxoplasmosis: a systematic review. Bull. World. Health. Organ. 91, 501-508.

476 Torrey, E.F., Bartko, J.J., Lun, Z.R., Yolken, R.H., 2007. Antibodies to Toxoplasma

477 gondii in patients with schizophrenia: a meta-analysis. Schizophr. Bull. 33, 729-

$478 \quad 736$.

479 Torrey, E.F., Yolken, R.H., 2001. The schizophrenia-rheumatoid arthritis connection:

480 infectious, immune, or both? Brain. Behav. Immun. 15, 401-410.

481 Torrey, E.F., Yolken, R.H., 2003. Toxoplasma gondii and schizophrenia. Emerg.

$482 \quad$ Infect. Dis. 9, 1375-1380.

483 Turney, K., 2012. Pathways of disadvantage: Explaining the relationship between

484 maternal depression and children's problem behaviors. Soc. Sci. Res. 41, 1546-

$485 \quad 1564$.

486 Wang, L., Wu, T., Anderson, J.L., Florence, J.E., 2011. Prevalence and risk factors of 487 maternal depression during the first three years of child rearing. J. Womens. 
489 Ye, H., Zou, P., 1993. Analysis of Toxoplasma gondii infection in 251 puerperae.

$490 \quad$ Chinese Journal of Zoonoses. 9, 64. (In Chinese)

491

492 
Table 1. Detection of anti-Toxoplasma gondii IgM and anti-T. gondii IgG antibodies in new mothers with baby blue and in the control group.

494

\begin{tabular}{ccccccc}
\hline & \multicolumn{2}{c}{ Toxoplasma gondii IgM } & & \multicolumn{3}{c}{ Toxoplasma gondii IgG } \\
\cline { 2 - 3 } \cline { 5 - 6 } \cline { 5 - 6 } & Total & & Total & postpartum blues & Normal \\
\hline No. examined & 475 & 475 & & 4706 & 1069 \\
No. positive & 0 & & & 27 & 7 & 20
\end{tabular}

495

Prevalence $(95 \% \mathrm{CI}), \%$

0

5.68(3.59-7.77) 6.60(1.80-11.41) 5.42(3.10-7.74)

OR $(95 \% \mathrm{CI})$

-

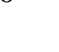

$\begin{array}{rcc}5.68(3.59-7.77) & 6.60(1.80-11.41) & 5.42(3.10-7.74) \\ -\quad \text { Ref. } & 1.234[0.507-3.002]\end{array}$

$P-\quad-\quad-0.64$

valu 3

497

498

499

500

501 
505 Table 2. Association between age, seropositivity of Toxoplasma gondii and prevalence of post-partum blues in new mothers. * Figures in

506 brackets indicate the number of cases of new mothers with both depression and T. gondii seropositivity.

507

$$
\begin{aligned}
& \text { Age Toxo post- } \\
& \text { plas partu } \\
& \text { ma } \mathrm{m} \\
& \text { gond blues } \\
& i i \\
& \mathrm{IgG}
\end{aligned}
$$

508

No. No. Prev OR $P$ - No. No. Prev OR $P$ -

exampositialenc ( $95 \%$ value exampositialenc $(95 \%$ value

ined ve e CI) ined ve e CI)

(case $(95 \%$

s $\mathrm{CI}), \quad \mathrm{CI})$,

with $\% \quad \%$

depre

ssion

*) 
509

$\begin{array}{lll}<5 & 57 \quad 2(0) 3.51 \text { ( Ref. } 0.872 \quad 57 \quad 14 & 24.56 \text { Ref. } 0.610\end{array}$

\begin{tabular}{|c|c|c|c|c|}
\hline & & $0-$ & & (13.0 \\
\hline & & 8.43) & & $4-$ \\
\hline & & & & 36.08 \\
\hline & & & & ) \\
\hline 25- 274 & 17 & $6.20(1.819$ & $274 \quad 63$ & 22.990 .917 \\
\hline 30 & (5) & $3.33-(0.40$ & & (17.9)(0.47 \\
\hline & & 9.08) 8- & & 8- $1-$ \\
\hline & & 8.102 & & 28.011 .784 \\
\hline
\end{tabular}

511

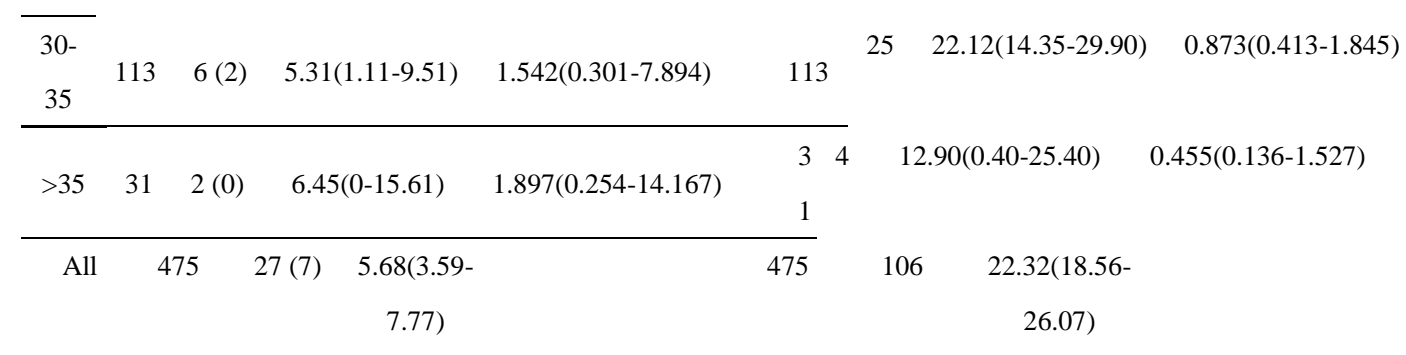

512 
519 Table 3. Occupation demographics associated with seropositivity of Toxoplasma gondii and prevalence of postpartum blues in new mothers.

520

Occupation Toxoplasma gondii $\operatorname{IgG}$ post-partum blues
No. No. Prevalence $(95 \% \mathrm{OR}(95 \%$ P- No
No. Prevalence $(95 \% \mathrm{OR}(95 \%$ P-
examinedpositive
$\mathrm{Cl}) \%$
CI) valueexaminedpositive
$\mathrm{CI}) \%$
CI) value

521

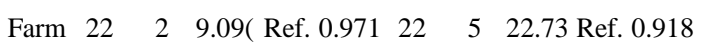

$(3.71$

22.14

) 41.75

522

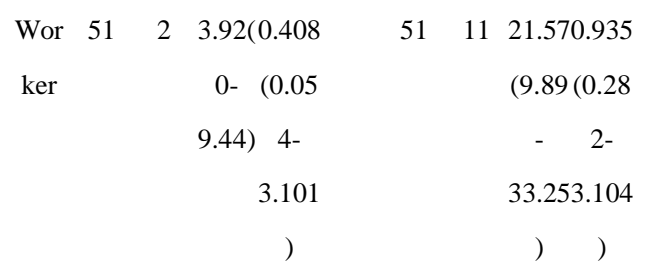




\begin{tabular}{|c|c|c|c|c|}
\hline Medi 36 & $\begin{array}{ll}2 & 5.56(0.588\end{array}$ & 36 & 6 & 16.670 .680 \\
\hline cal & $0-\quad(0.07$ & & & $(3.88(0.18$ \\
\hline staff & $13.427-$ & & & $-\quad 0-$ \\
\hline & ) 4.507 & & & 29.462 .565 \\
\hline
\end{tabular}

524

\begin{tabular}{|c|c|c|c|c|c|c|c|c|}
\hline Manager & 14 & 1 & $7.14(0-22.57)$ & $0.769(0.063-9.371)$ & 14 & 2 & $14.29(0-35.25)$ & $0.567(0.094-3.423)$ \\
\hline Teacher & 18 & & $5.56(0-$ & $0.588(0.049-$ & 18 & 4 & $22.22(0.95-$ & $0.971(0.218-$ \\
\hline & & & 17.28) & 7.067) & & & $43.50)$ & $4.323)$ \\
\hline
\end{tabular}

525

\begin{tabular}{|c|c|c|c|}
\hline Busi 66 & $34.55(0.476$ & 66 & $14 \quad 21.210 .915$ \\
\hline ness & $0-\quad(0.07$ & & (11.0) $(0.28$ \\
\hline staff & 9.71) 4- & & $9-\quad 7-$ \\
\hline & 3.055 & & 31.342 .916 \\
\hline & ) & & ) \\
\hline
\end{tabular}

526

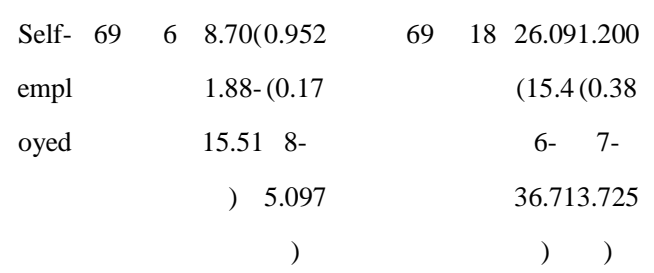

527
$\begin{array}{llll}\text { Hous } & 7 & 0 & 0.00(0.547\end{array}$
$\begin{array}{lll}7 & 3 & 42.862 .550\end{array}$
ewiv $\quad 0.00-(0.02$
(0- $(0.42$
es $\quad 0.00) \quad 3$
$92.292-$ 
$12.75 \quad 15.40$

$\left.0)^{*}-6\right)$

528

\begin{tabular}{|c|c|c|c|c|}
\hline Une 47 & $\begin{array}{ll}3 & 6.38(0.682\end{array}$ & 47 & 9 & 19.150 .805 \\
\hline mplo & $0-\quad(0.10$ & & & (7.47) 0. \\
\hline yed & $13.646-$ & & & - $4-$ \\
\hline & ) 4.405 & & & 30.832 .7 \\
\hline
\end{tabular}

529

Othe $145 \quad 7 \quad 4.83(0.507$

rs $\quad 1.30-(0.09 \quad(16.4(0.3)$

8.36) 8- 7- 8-

$2.615 \quad 30.433 .032$

530

531

532

536 Table 4. Risk factors associated with the seropositivity of Toxoplasma gondii and prevalence of postpartum blues in new mothers. 
537

Ris Tox

k opla

fact sma

or gon

dii

$\mathrm{IgG}$

538

No. examined $\quad$ No. positive (with depression) $\quad$ Prevalence(95\% CI),\% OR(95\% CI) $\quad P$-value

$\begin{array}{lllllll}\text { Cat Contact } & \text { No } & 380 & 21(4) & 5.53(3.22-7.83) & \text { Ref. } & 0.766\end{array}$

539

\begin{tabular}{lcrrrrr}
\hline & Yes & 95 & $6(3)$ & $6.32(1.33-11.3)$ & $1.152(0.452-2.940)$ & - \\
\hline Raw Meat & No & 384 & $20(2)$ & $5.21(2.98-7.44)$ & Ref. & 0.357
\end{tabular}

540

Yes $917(5) 7.691 .51$

$(2.17(0$.

13.2 -

7) 3.70

4)

541

542 
Figure legends.

551

552 Figure S1A. Quantitative comparison between Toxoplasma gondii immunoglobulin

553 (IgG) optical density (OD) and Hamilton Depression Scale (HAMD) score in all new

554 mothers $(\mathrm{n}=475)$. There is no significant correlation between IgG optical density and

555 HAMD score $(p=0.872)$.

556

557 Figure S1B. Quantitative analysis of all Toxoplasma gondii seropositive new mothers

558 ( $\mathrm{n}=27)$ by comparison of immunoglobulin ( $\operatorname{IgG})$ optical density (OD) and Hamilton

559 Depression Scale (HAMD) score in the same mothers. There is no significant

560 correlation between IgG optical density and HAMD score $(p=0.214)$.

561

562

563 
